Chapter 2

\title{
Scaling the Benefits of Digital Nonlinear Compensation in High Bit-Rate Optical Meshed Networks
}

\author{
Danish Rafique and Andrew D. Ellis \\ Additional information is available at the end of the chapter \\ http://dx.doi.org/10.5772/52743
}

\section{Introduction}

The communication traffic volume handled by trunk optical transport networks has been increasing year by year [1]. Meeting the increasing demand not only requires a quantitative increase in total traffic volume, but also ideally requires an increase in the speed of individual clients to maintain the balance between cost and reliability. This is particularly appropriate for shorter links across the network, where the relatively high optical signal-to-noise ratio (OSNR) would allow the use of a higher capacity, but is less appropriate for the longest links, where products are already close to the theoretical limits [2]. In such circumstances, it is necessary to maximize resource utilization and in a static network one approach to achieve this is the deployment of spectrally efficient higher-order modulation formats enabled by digital coherent detection. As attested by the rapid growth in reported constellation size $[3,4]$, the optical hardware for a wide variety of coherently detected modulation formats is identical [5]. This has led to the suggestion that a common transponder may be deployed and the format adjusted on a link by link basis to either maximize the link capacity given the achieved OSNR, or if lower, match the required client interface rate [6] such that the number of wavelength channels allocated to a given route is minimized. It is believed that such dynamic, potentially self-adjusting, networks will enable graceful capacity growth, ready resource re-allocation and cost reductions associated with improved transponder volumes and sparing strategies. However additional trade-offs and challenges associated with such networks are presented to system designers and network planners. One such challenge is associated with the nonlinear transmission impairments which strongly link the achievable channel reach for a given set of modulation formats, symbol-rates [6,7] across a number of channels. 
Various methods of compensating fiber transmission impairments have been proposed, both in optical and electronic domain. Traditionally, dispersion management was used to suppress the impact of fiber nonlinearities [8,9]. Although dispersion management is appreciably beneficial, the benefit is specific to a limited range of transmission formats and rates and it enforces severe limitations on link design. Similarly, compensation of fiber impairments based on spectral inversion (SI) [10], has been considered attractive because of the removal of in-line dispersion compensation modules (DCM), transparency to modulation formats and compensation of nonlinearity. However, although SI has large bandwidth capabilities, it often necessitates precise positioning and customized link design (e.g., distributed Raman amplification, etc.). Alternatively, with the availability of high speed digital signal processing (DSP), electronic mitigation of transmission impairments has emerged as a promising solution. As linear compensation methods have matured in past few years [11], the research has intensified on compensation of nonlinear impairments. In particular, electronic signal processing using digital back-propagation (DBP) with time inversion has been applied to the compensation of channel nonlinearities $[12,13]$. Back-propagation may be located at the transmitter [14] or receiver [15], places no constraints on the transmission line and is thus compatible with the demands of an optical network comprising multiple routes over a common fiber platform. In principle this approach allows for significant improvements in signal-to-noise ratios until the system performance becomes limited only by non-deterministic effects [16] or the power handling capabilities of individual components. Although the future potential of nonlinear impairment compensation using DBP in a dynamic optical network is unclear due to its significant computational burden, simplification of nonlinear DBP using single-channel processing at the receiver suggest that the additional processing required for intra-channel nonlinearity compensation may be significantly lower than is widely anticipated $[17,18]$. Studies of the benefits of DBP have largely been verified for systems employing homogenous network traffic, where all the channels have the same launch power [19]. However, as network upgrades are carried out, it is likely that channels employing different multi-level formats will become operational. In such circumstances, it has been demonstrated that the overall network capacity may be increased if the network traffic will become inhomogeneous, not only in terms of modulation format, but also in terms of signal launch power $[6,7,20]$. In particular, if each channel operates at the minimum power required for error free propagation (after error correction) rather than a global average power or the optimum power for the individual channel, the overall level of cross phase modulation in the network is reduced [20].

In this chapter we demonstrate the application of electronic compensation schemes in a dynamic optical network, focusing on adjustable signal constellations with non identical launch powers, and discuss the impact of periodic addition of 28-Gbaud polarization multiplexed m-ary quadrature amplitude modulation (PM-mQAM) channels on existing traffic. We also discuss the impact of cascaded reconfigurable optical add-drop multiplexerson networks operating close to the maximum permissible capacity in the presence of electronic compensation techniques for a range of higher-order modulation formats and filter shapes. 


\section{Simulation conditions}

Figure 1 illustrates the simulation setup. The optical link comprised nine (unless mentioned otherwise) 28-Gbaud WDM channels, employing PM-mQAM with a channel spacing of 50 GHz. For all the carriers, both the polarization states were modulated independently using de-correlated $2^{15}$ and $2^{16}$ pseudo-random bit sequences (PRBS), for $x$ - and y-polarization states, respectively. Each PRBS was de-multiplexed separately into two multi-level output symbol streams which were used to modulate an in-phase and a quadrature-phase carrier. The optical transmitters consisted of continuous wave laser sources, followed by two nested Mach-Zehnder Modulator structures for $\mathrm{x}$ - and $\mathrm{y}$-polarization states, and the two polarization states were combined using an ideal polarization beam combiner. The simulation conditions ensured 16 samples per symbol with $2^{13}$ total simulated symbols per polarization. The signals were propagated over standard single mode fiber (SSMF) transmission link with 80 $\mathrm{km}$ spans, no inline dispersion compensation and single-stage erbium doped fiber amplifiers (EDFAs). The fiber had attenuation of $0.2 \mathrm{~dB} / \mathrm{km}$, dispersion of $20 \mathrm{ps} / \mathrm{nm} / \mathrm{km}$, and a nonlinearity coefficient $(\gamma)$ of $1.5 / \mathrm{W} / \mathrm{km}$ (unless mentioned otherwise). Each amplifier stage was modeled with a $4.5 \mathrm{~dB}$ noise figure and the total amplification gain was set to be equal to the total loss in each span.

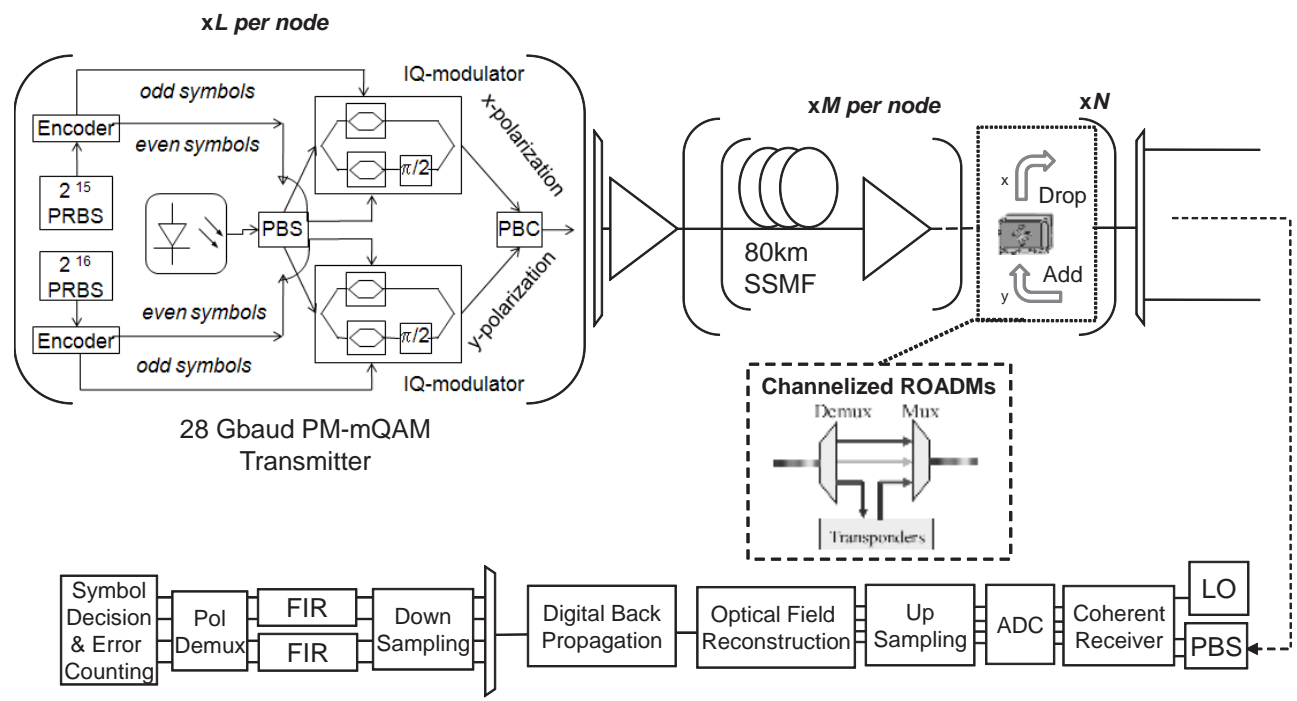

Figure 1. Simulation setup for 28-Gbaud PM-mQAM $(m=4,16,64,256)$ transmission system with $L$ wavelengths and $M$ spans per node (total spans is given by $N$ ).

At the coherent receiver the signals were pre-amplified (to a fixed power of $0 \mathrm{dBm}$ per channel), filtered with a $50 \mathrm{GHz} 3^{\text {rd }}$ order Gaussian de-multiplexing filter, coherently-detected and sampled at 2 samples per symbol. Transmission impairments were digitally compensat- 
ed in two scenarios. Firstly by using electronic dispersion compensation (EDC) alone, employing finite impulse response (FIR) filters (T/2-spaced taps) adapted using a least mean square algorithm. In the second case, electronic compensation was applied via single-channel digital back-propagation (SC-DBP), which was numerically implemented by split-step Fourier method based solution of nonlinear Schrödinger equation. In order to establish the maximum potential benefit of DBP, the signals were up sampled to 16 samples per bit and an upper bound on the step-size was set to be $1 \mathrm{~km}$ with the step length chosen adaptively based on the condition that in each step the nonlinear effects must change the phase of the optical field by no more than 0.05 degrees. To determine the practically achievable benefit, in line with recent simplification of DBP algorithms, e.g. [17,18,21], we also employed a simplified DBP algorithm similar to [21], with number of steps varying from $0.5 \mathrm{step} / \mathrm{span}$ to 2 steps/span. Following one of these stages (EDC or SC-DBP) polarization de-multiplexing, frequency response compensation and residual dispersion compensation was then performed using FIR filters, followed by carrier phase recovery [22]. Finally, the symbol decisions were made, and the performance assessed by direct error counting (converted into an effective $Q$-factor $\left(Q_{\text {eff }}\right)$ ). All the numerical simulations were carried out using VPItransmissionMaker®v8.5, and the digital signal processing was performed in MATLAB®V7.10.

\section{Analysis of trade-offs in hybrid networks}

\subsection{Constraints on transmission reach}

In a dynamic network, there are a large range of options to provide the desired flexibility including symbol rate [23], sub-carrier multiplexing [24], network configuration [25] signal constellation and various combinations of these techniques. In this section we focus on the signal constellation and discuss the impact of periodic addition of PM-mQAM (m=4, 16, 64, 256) transmission schemes on existing PM-4QAM traffic in a $28-G b a u d$ WDM optical network with a total transparent optical path of 9,600 km. We demonstrate that the periodic addition of traffic at reconfigurable optical add-drop multiplexer (ROADM) sites degrades through traffic, and that this degradation increases with the constellation size of the added traffic. In particular, we demonstrate that undistorted PM-mQAM signals have the greatest impact on the through traffic, despite such signals having lower peak-to-average power ratio (PAPR) than dispersed signals, although the degradation strongly correlated to the total PAPR of the added traffic at the launch point itself. Using this observation, we propose the use of linear pre-distortion of the added channels to reduce the impact of the cross-channel impairments [26,27].

Note that the total optical path was fixed to be $9,600 \mathrm{~km}$ and after every $M$ spans, a ROADM stage was employed and the channels to the left and right of the central channel were dropped and new channels with independent data patterns were added, as shown in Figure 2. in order to analyze the system performance, the dropped channels were coherently-detected after first ROADM and the central channel after the last ROADM link. 


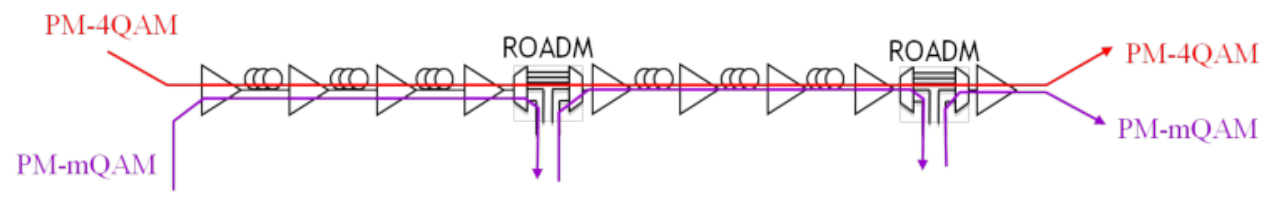

Figure 2. Network topology for flexible optical network, employing PM-4QAM traffic as a through channel, and PMmQAM traffic as neighboring channels, getting added/dropped at each ROADM site. Note that in this schematic only right-hand wavelength is shown to be added/dropped, however in the simulations both right and left wavelengths were add/dropped. The total path length was fixed to $9,600 \mathrm{~km}$, and the number of ROADMs was varied.

The optimum performance of the central PM-4QAM channel at 9,600 $\mathrm{km}$ occurred for a launch power of $-1 \mathrm{dBm}$. In this study, the launch power of all the added channels was also fixed at $-1 \mathrm{dBm}$, such that all channels had equal launch powers. Figure 3 illustrates the performance of the central test channel after the last node (solid), along with the performance of co-propagating channel employing various modulation formats after the first ROADM node (open) for a number of ROADM spacing's, using both single-channel DBP (Figure 3a) and EDC (Figure 3b). It can be seen that single-channel DBP offers a $\mathrm{Q}_{\text {eff }}$ improvement of $\sim 1.5 d B$ compared to EDC based system. This performance improvement is strongly constrained by inter-channel nonlinearities, such that intra-channel effects are not dominant. Moreover, the figure shows that as the number of ROADM nodes are increased, or the distance between ROADMs decreases, the performance of higher-order neighboring channels improves significantly due to the improved OSNR.

It can also be seen from Figure 3 that added channels with higher-order formats induce greater degradation of the through channel. In particular if there are 30 ROADM sites ( $320 \mathrm{~km}$ ROADM spacing) allocated to transmit PM-64QAM, whilst this traffic operates with significant margin, the through traffic falls below the BER of $3.8 \times 10^{-3}$. This increased penalty is due to the increased nonlinear degradation encountered in the first span after the ROADM node, where higher formats induce greater cross phase modulation(XPM) than PM-4QAM by virtue of their increased PAPR. However, even when the add drop traffic is PM-4QAM, the performance of the through channel degrades slightly as the number of ROADM nodes is increased, despite the reduction in PAPR due to the randomization of the nonlinear crosstalk.

The estimated PAPR evolutions for the various formats are shown in Figure 4. Asymptotic values are reached after the first span, and reach a slightly higher value for $m \geq 16$. The PAPR is reduced at the ROADM site itself, particularly for PM-4QAM. Figure 4 implies that harmful increases in the instantaneous amplitude of the interfering channels are not the entire cause of the penalty experienced by the through channel; we can therefore only conclude that the additional distortion results from interplay between channel walk off and nonlinear effects. Given that walk-off is known to induce short and medium range correlation in crosstalk between subsequent bits, effectively low pass filtering the crosstalk [28]. We thus believe that the penalty experienced by the through channel is not only because of variation in PAPR, but also due to the randomization of the crosstalk by the periodic replacement of the interfering data pattern. 

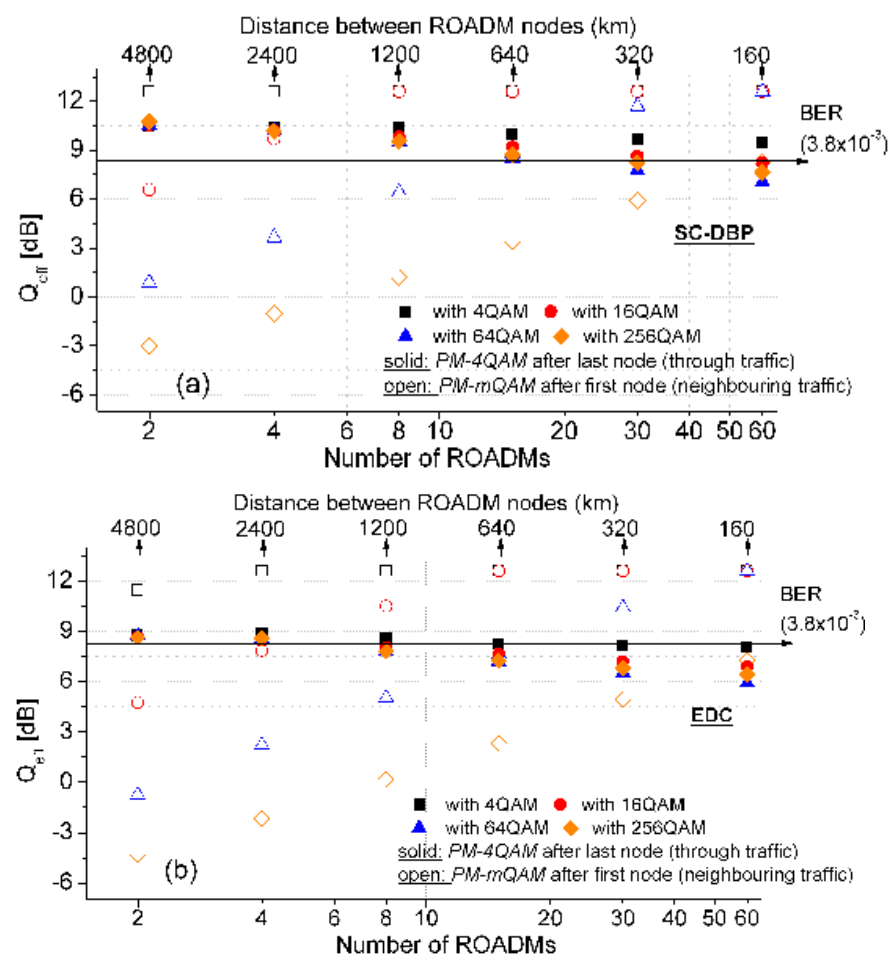

Figure 3. $Q_{\text {eff }}$ as a function of number of ROADMs (and distance between ROADM nodes) for 28-Gbaud PM-mQAM showing performance of central PM-4QAM (solid, after total length), and neighboring PM-mQAM (open, after first node). a) with single-channel DBP, b) with electronic dispersion compensation. Square: 4QAM, circle: 16QAM, up triangle: 64QAM, diamond:256QAM. Up arrows indicate that no errors were detected, implying that the $Q_{\text {eff }}$ was likely to be above $12.59 \mathrm{~dB}$. Total link length is $9,600 \mathrm{~km}$.

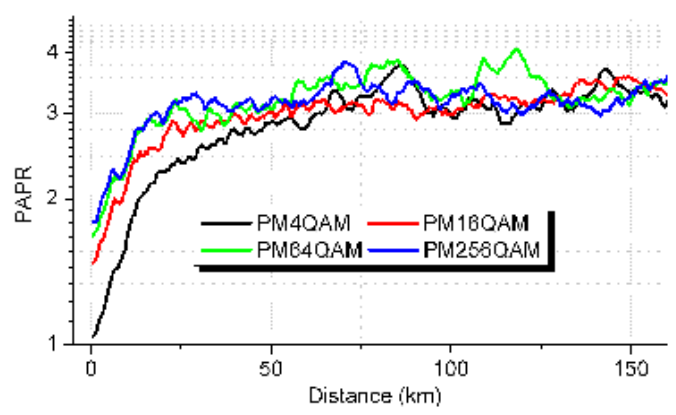

Figure 4. Variation in PAPR, for 4QAM (black), 16QAM (red), 64QAM (green) and 256QAM (blue) for a loss-less linear fiber with $20 \mathrm{ps} / \mathrm{nm} / \mathrm{km}$ dispersion. 


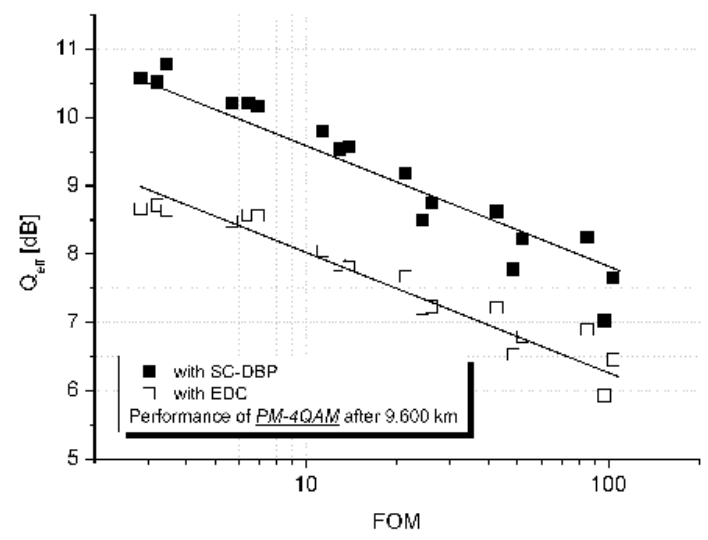

Figure 5. $Q_{\text {eff }}$ of the PM-4QAM through channel for 28-Gbaud PM-mQAM add/drop traffic after 9,600 km as a function of a figure of merit (FOM) defined in the text for various add drop configurations. Solid: with single-channel DBP, open: with EDC.

This is confirmed by Figure 5, which plots the $\mathrm{Q}_{\text {eff }}$ of PM-4QAM after last node, for both EDC and single-channel DBP, in terms of a figure of merit (FOM) related to the increased amplitude modulation experienced by the test channel in the spans immediately following the ROADM node, defined as,

$$
\operatorname{FOM}_{P M-m Q A M}(m)=\left(R O A D M_{N}\right) \times\left[I_{\text {max }}(m) / \overline{I_{\text {all }}(m)}\right]
$$

where $m$ represents the modulation order, $R O A D M_{N}$ represents number of add-drop nodes, $I_{\max }$ and $I_{\text {all }}$ are the maximum and mean intensity of the given modulation format at the ROADM site. A strong correlation between the penalty and change in PAPR is observed. For instance, for a high number of ROADMs the system would be mostly influenced by relatively un-dispersed signals and the difference between peak-to-average fluctuations for multi-order QAM varies significantly. This leads to higher-order modulation formats impinging worse cross-channel effects on existing traffic for shorter routes.

Having observed that the nonlinear penalty is determined by the reduction in the correlation of nonlinear phase shift between bits arising from changing bit patterns, and to changes in PAPR arising from undistorted signals, it is possible to design a mitigation strategy to minimize these penalties. Figure 6 illustrates, for both EDC and single-channel DBP systems, that if the co-propagating higher-order QAM channels are linearly pre-dispersed, the performance of the PM-4QAM through traffic can be improved. The figure shows that when positive pre-dispersion is applied, such that the neighboring channel constellation is never, along its entire inter node transmission length, restored to a well-formed shape, the impact of cross-channel impairments on existing traffic is reduced significantly. 


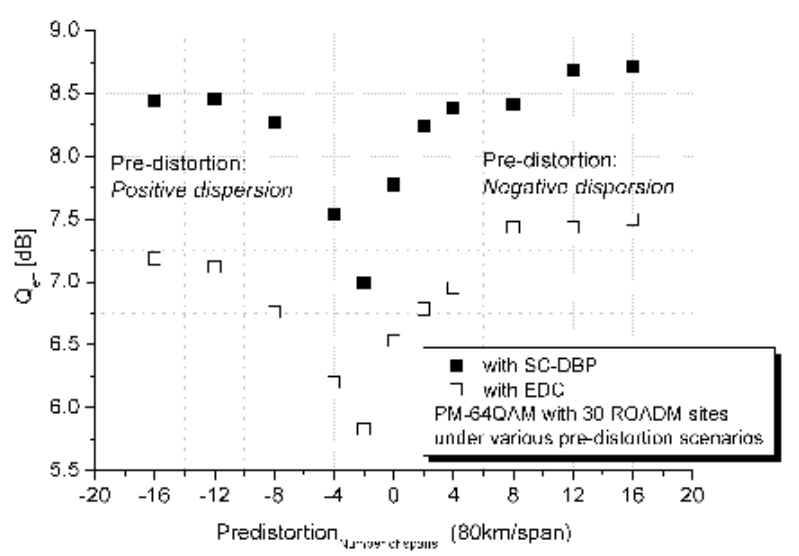

Figure 6. $\mathrm{Q}_{\text {eff }}$ of the PM-4QAM through channel with 30 ROADM sites, when the neighboring PM-64QAM channel is linearly pre-dispersed. Solid: with single-channel DBP, open: with EDC.

On the other hand, when negative pre-dispersion of less than the node-length (distance per node) is employed, the central test channel is initially degraded further. This behavior can be attributed to the increased impact of the PAPR of the un-dispersed constellation which is restored in the middle of the link. However, if negative pre-dispersion of more than the node-length is employed, the penalty is reduced due to lower PAPR induced XPM, and the performance saturates for higher values of pre-dispersion, similar to the case of positive predispersion. Note that avoiding well formed signals along the entire link corresponds to maximizing the path averaged PAPR of the signals. The benefits of this strategy have subsequently been predicted from a theoretical standpoint $\left.{ }^{[27}\right]$.

\subsection{Constraints on transmitted power}

In this section, we demonstrate that independent optimization of the transmitted launch power enhances the performance of higher modulation order add-drop channels but severely degrades the performance of through traffic due to strong inter-channel nonlinearities. However, if an altruistic launch power policy is employed such that the higher-order adddrop traffic still meets the BER of $3.8 \times 10^{-3}$, a trade-off can be recognized between the performance of higher-order channels and existing network traffic enabling higher overall network capacity with minimal crosstalk [19].

As a baseline for this study, we initially consider transmission distances up to $9,600 \mathrm{~km}$ with the same $80 \mathrm{~km}$ spans, suitable to enable a suitable performance margin (at bit-error rate of $3.8 \times 10^{-3}$ ) for the network traffic given various modulation schemes at a fixed launch power of $-1 \mathrm{dBm}$, (optimum power as determined in previous section. For a dynamic network with $N$ ROADMs and $m^{\text {th }}$ order PM-QAM, the overall results are summarized in Table 1 . The table shows under which conditions the central PM-4QAM channel (right-hand symbol), and the periodically added traffic (left-hand symbol) are simultaneously able to achieve errorfree operation after FEC. Two ticks indicate that both types of traffic is operational, whilst a 
cross indicates that at least one channel produces severely errorred signals. As expected, with decreasing ROADM spacing, the operability of higher-order neighboring channels increases due to the improved OSNR. However, it can also be seen that as a consequence, added channels with higher-order formats induce greater degradation of the through channel through nonlinear crosstalk as shown in Section 3.1. In particular, if the ROADM spacing is $320 \mathrm{~km}$, allocated to transmit PM-64QAM, whilst this traffic is operable, the through traffic falls below the BER threshold. Conversely for large ROADM spacing, there is little change in nonlinear crosstalk, since the m-QAM signals are highly dispersed, but the higher order format traffic has insufficient OSNR for error free operation. We refer to this approach as "fixed network power".

\begin{tabular}{|c|c|c|c|c|c|c|c|}
\hline $\begin{array}{c}\text { mQAM/ } \\
\text { ROADM spacing }\end{array}$ & $\begin{array}{l}4800 \\
\mathrm{~km}\end{array}$ & $\begin{array}{c}2400 \\
\mathrm{~km}\end{array}$ & $\begin{array}{l}1200 \\
\mathrm{~km}\end{array}$ & $\begin{array}{l}640 \\
\mathrm{~km}\end{array}$ & $\begin{array}{l}320 \\
\mathrm{~km}\end{array}$ & $\begin{array}{l}160 \\
\mathrm{~km}\end{array}$ & $\begin{array}{l}80 \\
\mathrm{~km}\end{array}$ \\
\hline 4QAM & ++ & ++ & ++ & ++ & ++ & ++ & ++ \\
\hline 16QAM & $x+$ & ++ & ++ & ++ & ++ & $+x$ & $+x$ \\
\hline 64QAM & $x+$ & $x+$ & $x+$ & ++ & $+x$ & $+x$ & $+x$ \\
\hline 256QAM & $x+$ & $x+$ & $x+$ & $x+$ & $x x$ & $x x$ & $+x$ \\
\hline
\end{tabular}

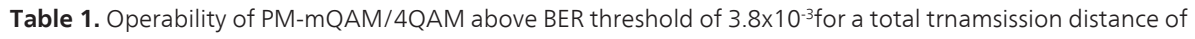
$9,600 \mathrm{~km}$. Tick/Cross (Left) represents performance of mQAM, Tick/Cross (Right) represents corresponding performance of central 4QAM. Tick: Operational, Cross: Non-operational

Since higher-order modulation formats have higher required OSNR, we expect the optimum launch power for those channels to be different than those used in the fixed network power scenario which was operated at a launch power of $-1 \mathrm{dBm}$. Thus, for example, for large ROADM spacing, we improved performance might be expected if the add-drop traffic operates with increased launch power. Figure 7 illustrates the performance of through channel and the higher-order add-drop channels as a function of launch power of the add-drop traffic (through channel operates with a fixed, previously optimized, launch power of $-1 \mathrm{dBm}$ ). For clarity we report two ROADM spacings, selected to give zero margin (Figure 7a) or $~ 2$ $d B$ margin (Figure $7 \mathrm{~b}$ ) for 256QAM add drop traffic. The ROADM spacing for 16 and 64QAM signals were scaled in proportion (approximately) to their required OSNR levels under linear transmission. The exact ROADM spacing is reported in the figure captions.

Figure 7 clearly illustrates that the higher-order formats operating over a longer (shorter) reach enable lower (higher) $Q_{\text {eff }}$ but also that the nonlinear effects increase in severity as the modulation order is increased. In particular, the long distance through traffic is strongly degraded before the nonlinear threshold is reached for such formats. Comparing Figure 7a and Figure 7b, we can see that the reduced ROADM spacing in Figure $7 \mathrm{~b}$ enables improved performance of the add-drop channels; however the degradation of the through channel is increasingly severe. This change in behavior between formats can be attributed to the increased amplitude modulation imposed by un-dispersed signals added at each ROADM site, as discussed previously. 

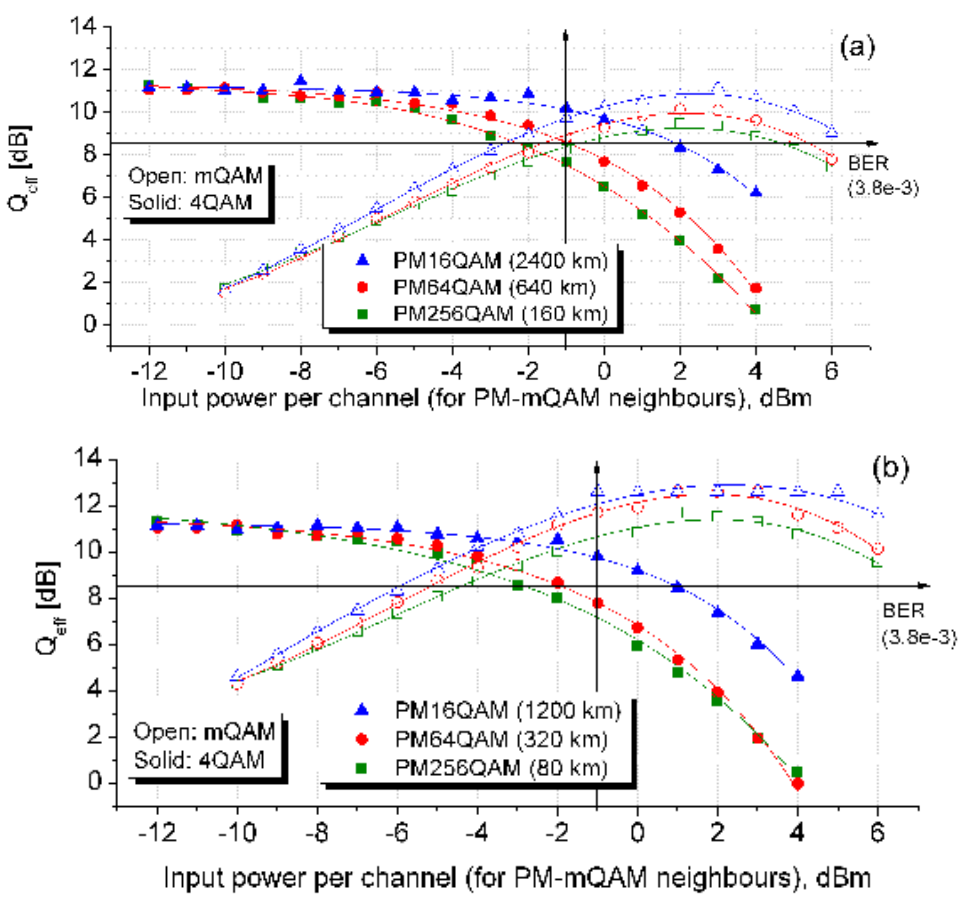

Figure 7. $Q_{\text {eff }}$ as a function of launch power of two neighboring channels for 28-Gbaud PM-mQAM, showing performance of central PM-4QAM (Solid), and neighboring PM-mQAM (Half Solid). Triangle: 16QAM, Circle: 64QAM, Square: 256QAM. The launch power per channel for PM-4QAM is fixed to $-1 \mathrm{dBm}$. ROADM spacing of, a) 2400,640, 160 km, b) $1200,320,80 \mathrm{~km}$ for 16, 64, 256 QAM, respectively.

We can use the results of Figure 7 to analyze the impact of various power allocation strategies. Clearly if we allow each transponder to adjust its launch power to optimize its own performance autonomously, a high launch power will be selected and the degradation to the traffic from other transponders increases in severity, and in all six scenarios in Figure 7 the through channel fails if the performance of the add drop traffic is optimized independently. This suggests that launch power should be centrally controlled. Howevercentrally controlled optimization of individual launch powers for each transponder is complex; so a more promising approach would be a fixed launch power irrespective of add-drop format or reach to minimize the complexity of this control. We have already seen (Table 1) that if the launch power is set to favor the performance of PM-4QAM $(-1 \mathrm{dBm})$ the flexibility in transmitted format for the add/drop transponders is low, and to confirm this in Figure 7 four of the scenarios fail. The best performance for these two scenarios is achieved at a fixed launch power of $-3 \mathrm{dBm}$, but we still find that 3 scenarios fail to establish error free connections. However, if the transponders are altruistically operated at the minimum launch power required for the desired connection (not centrally controlled), the majority of the scenarios studied result in successful connections. The one exception is the add-drop of 256QAM 
channels with a ROADM spacing of $160 \mathrm{~km}$, which is close to the maximum possible reach of the format. Note that shorter through paths would tend to use higher-order formats for all the routes, where nonlinear sensitivity is higher [29], and therefore we expect similar conclusions.

\section{Application in meshed networks}

In the previous section, we identified that optimum performance for a given predetermined modulation format was obtained by using the minimum launch power. However, this arbitrary selection of transmitted format fails to take into account the ability of a given link to operate with different formats, leading to a rich diversity of connections. In this section, we focus on the impact of flexibility in the signal constellation, allowing for evolution of the existing ROADM based static networks. We consider a configuration where network capacity is increased by allowing higher-order modulation traffic to be transmitted on according to predetermined rules based on homogenous network transmission performance. In particular we consider a $50 \mathrm{GHz}$ channel grid with coherently-detected 28-Gbaud PM-mQAMand 20 wavelength channels. We demonstrate that even if modulation formats are chosen based on knowledge of the maximum transmission reach aftersingle-channel digital back-propagation, for the network studied, the majority of the network connections (75\%) are operable with significant optical signal-to-noise ratio margin when operated with electronic dispersion compensation alone. However, $23 \%$ of the links require the use of single-channel DBP for error free operation. Furthermore, we demonstrate that in this network higher-order modulation formats are more prone to impairments due to channel nonlinearities and filter crosstalk; however they are less affected by the bandwidth constrictions associated with ROADM cascades due to shorter operating distances. Finally, we show that, for any given modulation order, a minimum filter Gaussian order of $\sim 3$ or bandwidth of $\sim 35 \mathrm{GHz}$ enables the performance with approximately less than $1 \mathrm{~dB}$ penalty with respect to ideal rectangular filters [30].

\subsection{Network design}

To establish a preliminary estimate of maximum potential transmission distance of each available format, we employed the transmission reaches identified in Section 3. These are suitable to enable a BER of $3.8 \times 10^{-3}$ at a fixed launch power of $-1 \mathrm{dBm}$ assuming the availability of single-channel DBP. These conditions gave maximum reaches of $2,400 \mathrm{~km}$ for PM-16QAM, $640 \mathrm{~km}$ for PM-64QAM and $160 \mathrm{~km}$ for 256 QAM. Note that only single-channel DBP was considered in this study since in a realistic mesh network access to neighboring traffic might be impractical. WDM based DBP solution may be suitable for a point to point submarine link or for a network connection where wavelengths linking the same nodes copropagate using adjacent wavelengths. Implementation of this condition would require DBP aware routing and wavelength assignment algorithms. This approach could enable significant $\mathrm{Q}_{\text {eff }}$ improvements or reach increases. For 64QAM, up to $7 d B Q_{\text {eff }}$ improvements were shown in $\left[{ }^{29}\right]$, although the benefit depends on the number of processed channels [31]. 
We then applied this link capacity rule to an 8-node route from a Pan-European network topology (see highlighted link in Figure 8). To generate a representative traffic matrix, for each node, commencing with London, we allocated traffic demand from the node under consideration to all of the subsequent nodes, operating the link at the highest order constellation permissible for the associated transmission distance, and selecting the next wavelength. We note that none of the links in this chosen route were suitable for 256QAM, indeed only the Strasberg to Zurich and Vienna to Prague links are expected to be suitable for this format.

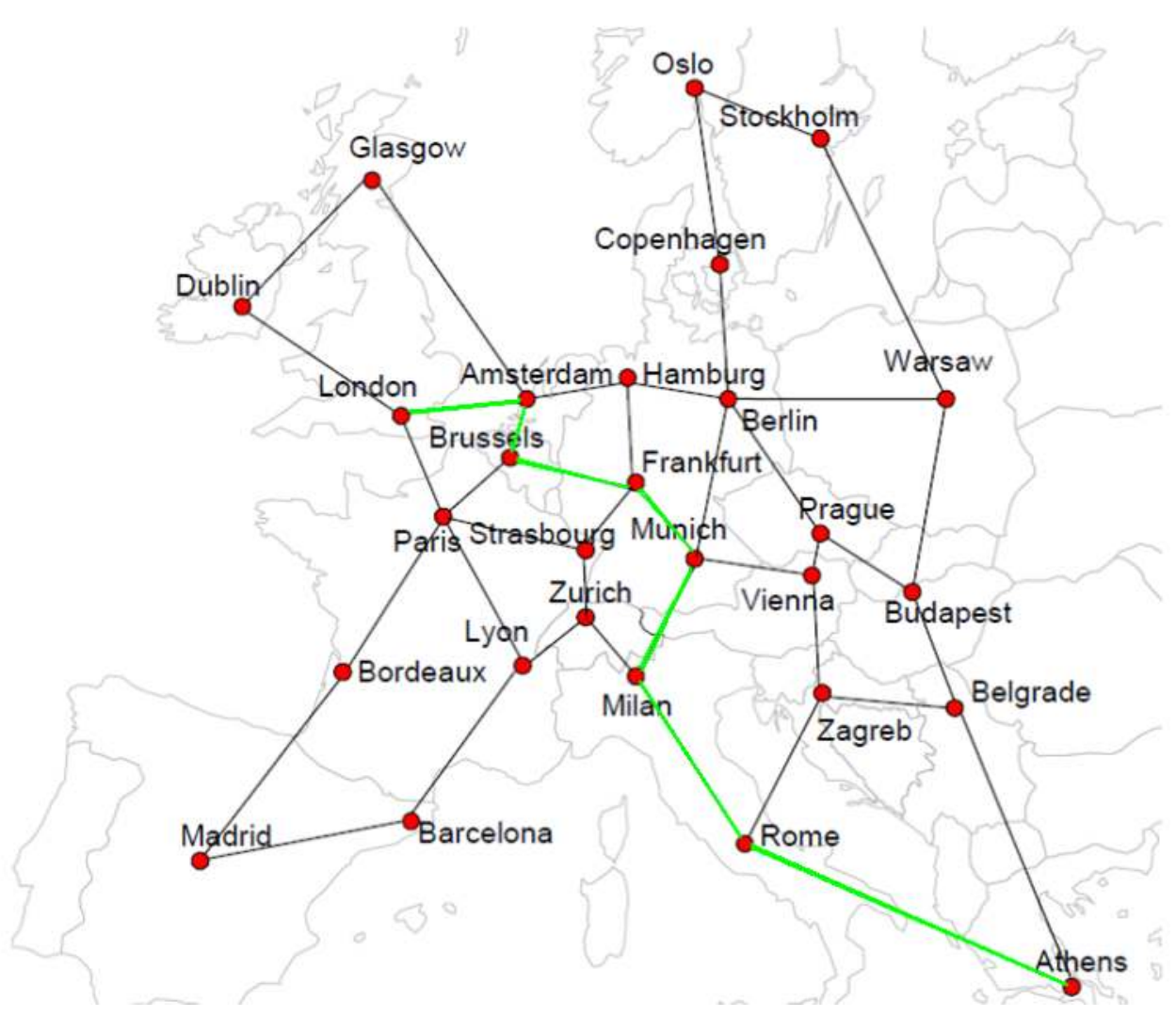

Figure 8. node Pan-European network topology. Link 1: London-to-Amsterdam: 7 spans, Link 2: Amsterdam-to-Brussels: 3 spans, Link 3: Brussels-to-Frankfurt: 6 spans. Link 4: Frankfurt-to-Munich: 6 spans, Link 5: Munich-to-Milan: 7 spans, Link 6: Milan-to-Rome: 9 spans, Link 7: Rome-to-Athens: 19 spans. (80 km/span).

Once all nodes were connected by a single link, this process was repeated (in the same order), adding additional capacity between nodes where an unblocked route was available until all 20 wavelengths were allocated, and no more traffic could be assigned without blockage. 
Table 2 illustrates the resultant traffic matrix showing the location where traffic was added and dropped (gray highlighting) and the order of the modulation format (numbers) carried wavelength (horizontal index) on each link (vertical index). For example, emerging from node 6 are nine wavelengths carrying PM-4QAM and 5 wavelengths carrying PM-16QAM whilst on the center wavelength, PM-16QAM data is transmitted from node 1 (London) to node 5 (Munich) where this traffic is dropped and replaced with PM-64QAM traffic destined for node 6 (Milan). This ensured that various nodes were connected by multiple wavelengths. As it can be seen, the adopted procedure allowed for a reasonably meshed optical network (36 connections) with shortest route of 3 spans and longest path of 57 spans, emulating a quasi-real traffic scenario with highly heterogeneous traffic. At each node, add-drop functionality was enabled using a channelized ROADM architecture where all the wavelengths were de-multiplexed and channels were added/dropped, before re-multiplexing the data signals again. We considered Rectangular and Gaussian-shaped filters for ROADM stages, and the order of the Gaussian filters was varied from 1 through 6.

\begin{tabular}{|c|c|c|c|c|c|c|c|c|c|c|c|c|c|c|c|c|c|c|c|c|}
\hline $\begin{array}{c}X \\
(\lambda) \\
Y \\
\text { (Link) }\end{array}$ & -10 & -9 & -8 & -7 & -6 & -5 & -4 & -3 & -2 & -1 & 0 & +1 & +2 & +3 & +4 & +5 & +6 & +7 & +8 & +9 \\
\hline 1 & 4 & 16 & 16 & & & & & 64 & 4 & 16 & 16 & 4 & 64 & & 16 & 16 & 16 & & 16 & 4 \\
\hline 2 & 4 & 16 & 16 & 16 & 64 & 64 & 4 & 16 & 4 & 16 & 16 & 4 & 16 & 4 & 16 & 16 & 16 & & 16 & 4 \\
\hline 3 & 4 & 16 & 16 & 16 & 4 & 16 & 4 & 16 & 4 & 16 & 16 & 4 & 16 & 4 & 16 & 16 & 16 & 64 & 16 & 4 \\
\hline 4 & 4 & 16 & 4 & 16 & 4 & 16 & 4 & 16 & 4 & 16 & 16 & 4 & 16 & 4 & 16 & 16 & 64 & 16 & 16 & 4 \\
\hline 5 & 4 & 16 & 4 & 16 & 4 & 16 & 4 & 16 & 4 & 16 & 64 & 4 & 16 & 4 & 4 & 16 & & 16 & & 4 \\
\hline 6 & 4 & & 4 & & 4 & & 4 & & 4 & 16 & 16 & 4 & 16 & 4 & 4 & 16 & & 16 & & 4 \\
\hline 7 & 4 & & 4 & & 4 & & 4 & & 4 & 16 & 16 & & & & 4 & & & & & \\
\hline
\end{tabular}

Table 2. Traffic matrix (Each element represents the modulation order, Grayed: Traffic dropped and added at nodes highlighted in gray.

\subsection{Results and discussions}

\subsubsection{Nonlinear transmission with ideal ROADMs}

Figure 9 depicts the required OSNR of each connection as a function of transmission distance, after electronic dispersion compensation. Note that in this case we employed rectangular ROADM filters to isolate the impact of inter-channel nonlinear impairments from filtering crosstalk (no cascade penalties were observed with ideal filters).

Numerous conclusions can be ascertained from this figure. First, these results confirm that with mixed-format traffic and active ROADMs, as the transmission distance is increased the required OSNR increases irrespective of the modulation order due to channel nonlinearities. 
Second, as observed by the greater rate of increase in required OSNR with distance, the higher-order channels are most degraded by channel nonlinearities, even at the shortest distance traversed. Furthermore, even for the shortest distances the offset between the theoretical OSNR for a linear system and the simulated values are greater for higher order formats. These two effectsare attributed to the significantly reduced minimum Euclidian distance which leads to increased sensitivity to nonlinear effects. However, for a system designed according to single-channel DBP propagation limits, as the one studied here, one can observe that majority of the links operate using EDC alone (except the ones highlighted by up-arrows). Note that managing the PAPR for such formats through linear pre-dispersion could further improve the transmission performance, as shown in Section 1.3. Additionally, in order to examine the available system margin, Figure 9 also shows the received OSNR for various configurations, where it can be seen that majority of the links (except 3 ) have more than $2 d B$ available margins, and that our numerical results show an excellent match to the theoretical predictions.

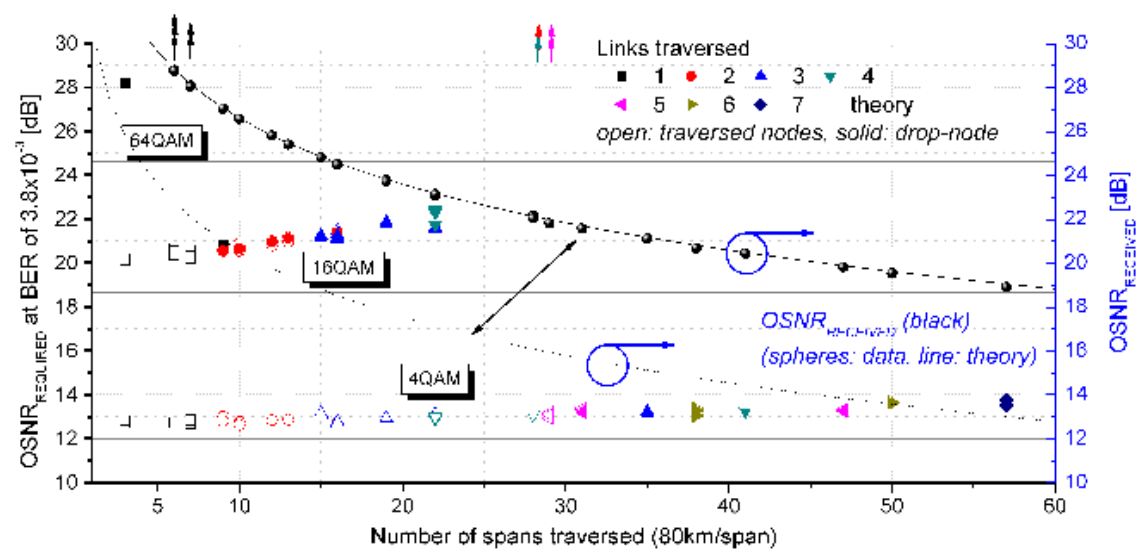

Figure 9. Nonlinear tolerance of PM-mQAM in a dynamic mesh network after EDC. a) Colored: OSNR at BER of 3.8 $\times 10^{-3}$ vs. Distance (Links traversed: 1(square), 2(circle), 3(up-tri), 4(down-tri), 5(left-tri), 6(right-tri), 7(diamond), horizontal lines (theoretical required OSNR)), open: intermediate nodes, solid: destination nodes. Black: Received OSNR (black spheres), Line (theoretical received OSNR), Dotted Line (theoretical received OSNR with $5 d B$ margin). Up arrows indicate failed connections (corresponding to drop nodes).

As discussed, the results presented in Figure 9 exclude 9 network connections classified as failed ( $25 \%$ of the total traffic), where the calculated BER was always found to be higher than the $3.8 \times 10^{-3}$. In order to address the failed routes, we employed single-channel DBP, as shown in [21], on such channels, as shown in Figure 10 (red: simplified, blue: full-precision 40 steps per span).It can be seen that all but one of the links can be restored by using singlechannel DBP, with the $\mathrm{Q}_{\text {eff }}$ increasing by an average of $\sim 1 \mathrm{~dB}$, consistent with the improvements observed for heterogeneous traffic in Section 1.3. The link which continues to give a BER even after after single-channel DBP is operated with the highest order modulation for- 
mat studied, and its two nearest neighbors are both highly dispersed. Note that even though the maximum node lengths are chosen based on nonlinear transmission employing singlechannel DBP, most of the network traffic also abide by the EDC constraints (64QAM: $\geq 1$ span, 16QAM: $\geq 6$ spans, 4QAM $\geq 24$ spans). The failed links have one-to-one correlation with violation of these EDC constraints, allowing for prediction of DBP requirements with a quarter of the total network traffic requiring the implementation of single-channel DBP. Also, note that all but two of the links are operable with less than 15 DBP steps for the whole link.

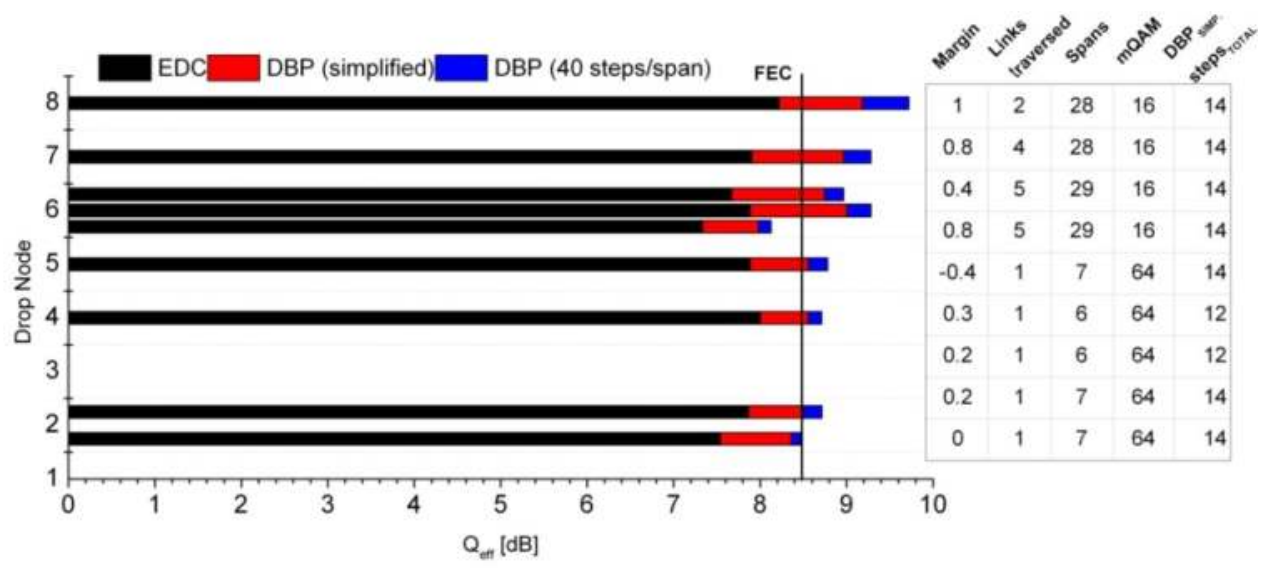

Figure 10. $Q_{\text {eff }}$ as a function of network nodes for failed routes, shown by up-arrows in Fig. 5, for PM-mQAM in a dynamic mesh network. After EDC (black) and single-channel DBP (red: simplified, blue: full-precision 40 steps per span). Table shows the network parameters for each scenario and number of steps for single-channel simplified DBP.

These results give some indication of the benefit of flexible formats and DBP. For particular network studied (assuming one of the two failed links works with high precision DBP), if homogeneous traffic, employing 4QAM, is considered, a total network capacity of 4-Tb/ scould be achieved. On the other hand, flexible m-ary QAM employing bandwidth allocation based on EDC performance limits only (not shown) enables $\sim 60 \%$ increase in transmission capacity $(6.8-\mathrm{Tb} / \mathrm{s})$, while designs accounting for SC-DBP add a further $12 \%$ increase in capacity $(7.7-T b / s)$. Note that for traffic calculations based on EDC constraints, we assumed that the routes of Figure 10 would operate satisfactorily for the next format down and that there would be no increase in the nonlinear penalty experienced by any other channel. Further increase in capacity can be attained if pre-dispersion or limited WDM DBP are used, or if more format granularity is introduced (e.g. 8QAM and 32QAM) to exploit the remaining margin. In this example, $25 \%$ of transponders operating in single-channel DBP mode enable a $12 \%$ increase in capacity. One may therefore argue that in order to provide a the same increase in capacity without employing DBP, approximately $12 \%$ more channels would be required, consuming 12\% more energy (assuming that the energy consumption is dominated by the transponders). In the case studied, since a $1 / 4$ of transponders require DBP, breakeven 
would occur if the energy consumption of a DBP transponder was $50 \%$ greater than a conventional transponder. Given that commercial systems allocate approximately 3-5\% of their power to the EDC chipset [32], this suggests that the DBP unit used could be up to 16 times the complexity of the EDC chip. The results reported in Figure 10 with simplified DBP fall within this bound and highlight the practicality of simplified DBP algorithms.
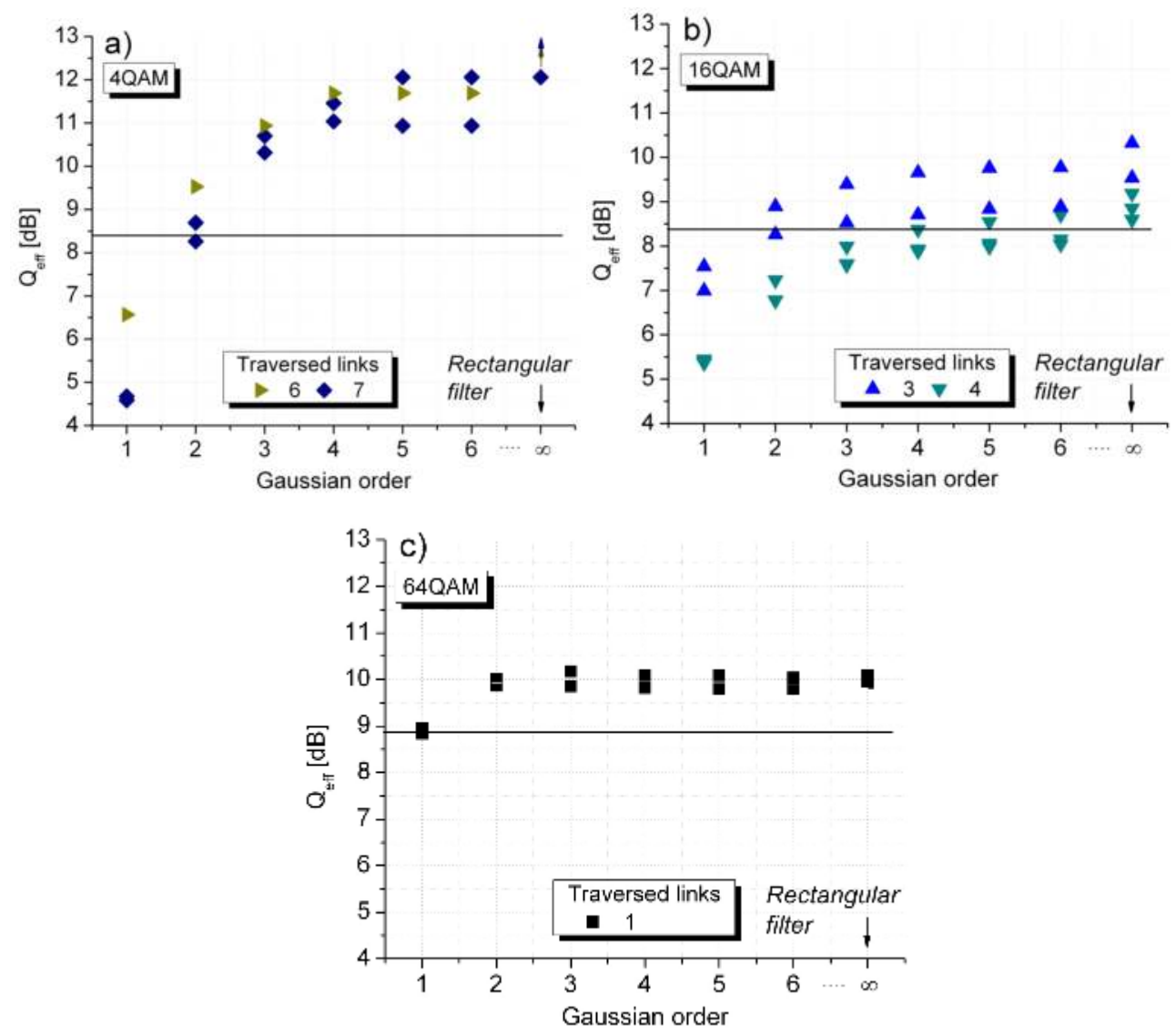

Figure 11. $Q_{\text {eff }}$ as a function of Gaussian filter order ( $35 \mathrm{GHz}$ bandwidth) for a $6 \mathrm{~dB}$ margin from theoretical achievable OSNR. a) 4QAM; b) 16QAM; c) 64QAM. (up-arrows indicate that no errors were detected).

\subsubsection{Filter order and $B W$ dependence}

Figure 11 shows the performance of a selection of links with less than $6 \mathrm{~dB}$ margin from the theoretical achievable OSNR (see Figure 9 for links used, we show only the links with the worst required OSNR in the case of 16QAM for clarity), as a function of the Gaussian filter order within each ROADM. As it is well-known, the transmission penalty decreases as filter 
order increases [33]. However, it can be seen that for higher-order modulation formats, the transmission performance saturates at lower filter orders, compared to lower-order formats. This trend is related to the fact that modulation formats traversing through greater number of nodes are more strongly dependent on the Gaussian order (attributed to known penalties from filter cascades [34,35]). For instance, the performance of 4QAM traffic is severely degraded as a function of Gaussian order, due to the higher number of nodes traversed by such format. 16QAM channels show relatively good tolerance to filter order due to reduced number of hops, however when greater than 3 nodes are employed, the performance again becomes a strong function of filter order. 64QAM is least dependent on filter order since no intermediate ROADMs are traversed. For any given modulation order, a minimum Gaussian order of $\sim 3$ enables the optimum performance to be within $1 \mathrm{~dB}$ of the performance for an ideal rectangular filter.
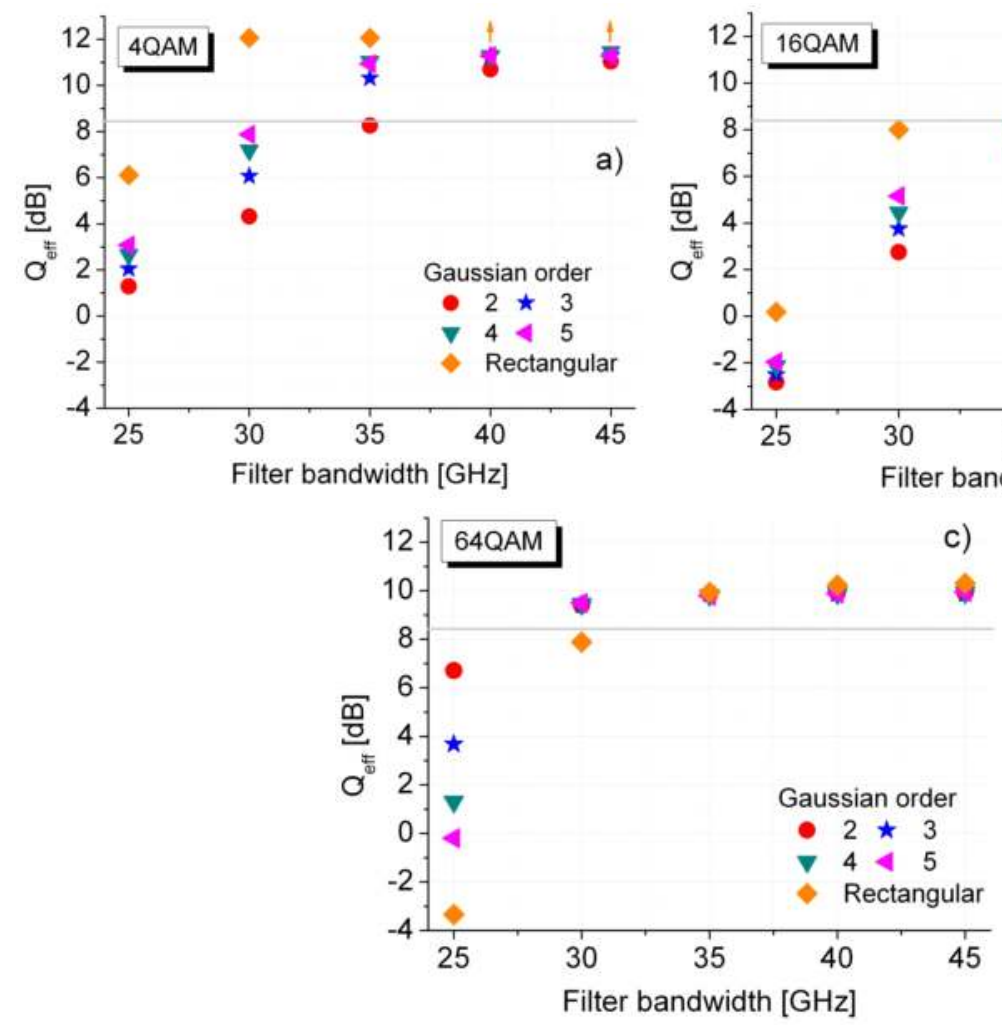

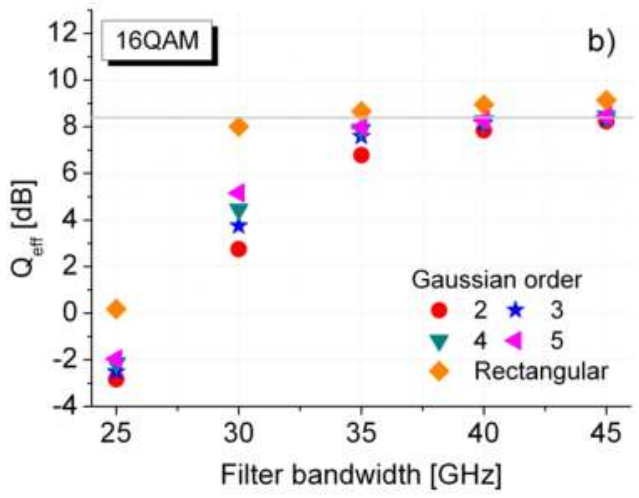

c)

Figure 12. $Q_{\text {eff }}$ as a function of Gaussian filter bandwidth (and filter order) for worst-case OSNR margin seen in Figure 6.8. a) 4QAM; b) 16QAM; c) 64QAM.

The simulated $Q_{\text {eff }}$ versus $3 d B$ bandwidth of the ROADM stages and filter order is shown in Figure 12, again for the worst-case required OSNR observed in Figure 9 for each modulation 
format. For lower bandwidths, the $\mathrm{Q}_{\text {eff }}$ is degraded due to bandwidth constraints. With the exception of second order filters, bandwidths down to $35 \mathrm{GHz}$ are sufficient for all the formats studied. However, consistent with previous analysis (in Figure 10), the impact of filter order on 64QAM is minimal and lower-order filters seem to have better performance than higher-order ones at $25 \mathrm{GHz}$ bandwidth. This is because when the signal bandwidth (28$\mathrm{GHz}$ ) exceeds the filter bandwidth, the lower order filters capture more of the signal spectra. However, this effect is visible in the case of 64QAM only since no nodes were traversed in this case, thereby avoiding the penalty from ROADM stages with lower filter orders.

\section{Summary and future work}

In this chapter we explored the network aspect of advanced physical layer technologies, including multi-level formats employing varying DSP, and solutions were proposed to enhance the capacity of static transport networks. It was demonstrated that that if the order of QAM is adjusted to maximize the capacity of a given route, there may be a significant degradation in the transmission performance of existing traffic for a given dynamic network architecture. Such degradations were shown to be correlated to the accumulated peak-toaverage power ratio of the added traffic along a given path, and that management of this ratio through pre-distortion was proposed to reduce the impact of adjusting the constellation size on through traffic. Apart from distance constraints, we also explored limitations in the operational power range of network traffic. The transponders which autonomously select a modulation order and launch power to optimize their own performance were reported to have a severe impact on co-propagating network traffic. A solution was proposed to operate the transponders altruistically, offering lower penalties than network controlled fixed power approach. In the final part of our analysis, the interplay between different higher-ordermodulation channels and the effect of filter shapes and bandwidth of(de)multiplexers on the transmission performance, in a segment of pan-European optical network was explored. It was verified that if the link capacities are assigned assuming that digital back propagation is available, $25 \%$ of the network connections fail using electronic dispersion compensation alone. However, majority of such links can indeed be restored by employing single-channel digital back-propagation. Our results indicated some benefit of flexible formats and DBP in realistic mesh networks. We showed that for particular network studied, if homogeneous traffic, employing 4QAM is considered, a total network capacity of $4 \mathrm{~Tb} / \mathrm{s}$ can be achieved. On the other hand, flexible m-ary QAM employing bandwidth allocation based on EDC performance limits enable $\sim 60 \%$ increase in transmission capacity $(6.8 \mathrm{~Tb} / \mathrm{s})$, while designs accounting for SC-DBP add a further $12 \%$ increase in capacity $(7.7 \mathrm{~Tb} / \mathrm{s})$. Further enhancement in network capacity may be obtained through the use of intermediate modulation order, dispersion pre-compensation for nonlinearity control and the use of altruistic launch powers.

In terms of network evolution, the ultimate goal is to enable software-defined transceivers, where each node would switch itself to just-right modulation scheme and associated DSP, based on various physical layer, distance, power, and etc. constraints. Modeling of real-time traffic employing the content covered in this chapter, should motivate and pave the way for 
high capacity upgrade of currently deployed networks. In addition, modulation/DSP aware routing and wavelength assignment algorithms (e.g. DBP bandwidth aware wavelength allocation) would further enhance the transmission capacity.

\section{Acknowledgements}

This work was supported by Science Foundation Ireland under Grant numbers 06/IN/I969 and 08/CE/11523.

\section{Author details}

Danish Rafique ${ }^{1,2}$ and Andrew D. Ellis ${ }^{1}$

1 Photonic Systems Group, Tyndall National Institute and Department of EE/Physics, University College Cork, Dyke Parade, Ireland

2 now with Nokia Siemens Networks, S.A., Lisbon, Portugal

\section{References}

[1] R.W. Tkach, "Scaling optical communications for the next decade and beyond," Bell Labs Technical Journal 14, 3-9 (2010).

[2] P. Winzer, “Beyond 100G Ethernet," IEEE Communications Magazine 48, 26 (2010).

[3] S. Makovejsm, D. S. Millar, V. Mikhailov, G. Gavioli, R. I. Killey, S. J. Savory, and P. Bayvel, "Experimental Investigation of PDMQAM16 Transmission at 112 Gbit/s over 2400 km," OFC/NFOEC, OMJ6 (2010).

[4] J. Yu, X. Zhou, Y. Huang, S. Gupta, M. Huang, T. Wang, and P. Magill, “112.8-Gb/s PM-RZ 64QAM Optical Signal Generation and Transmission on a $12.5 \mathrm{GHz}$ WDM Grid," OFC/NFOEC, OThM1 (2010).

[5] M. Seimetz, Higher-order modulation for optical fiber transmission. Springer (2009).

[6] A. Nag, M. Tornatore, and B. Mukherjee, “Optical network design with mixed line rates and multiple modulation formats," Journal of Lightwave Technology 28, 466475 (2010).

[7] C. Meusburger, D. A. Schupke, and A. Lord, "Optimizing the migration of channels with higher bitrates," Journal of Lightwave Technology 28, 608-615 (2010). 
[8] M. Suzuki, I. Morita, N. Edagawa, S. Yamamoto, H. Taga, and S. Akiba, "Reduction of Gordon-Haus timing jitter by periodic dispersion compensation in soliton transmission," Electronics Letters 31, 2027-2029 (1995).

[9] C. Fürst, C. Scheerer, G. Mohs, J-P. Elbers, and C. Glingener, "Influence of the dispersion map on limitations due to cross-phase modulation in WDM multispan transmission systems," Optical Fiber Communication Conference, OFC '01, MF4 (2001).

[10] D.D. Marcenac, D. Nesset, A. E. Kelly, M. Brierley, A. D. Ellis, D. G. Moodie, and C. W. Ford, "40 Gbit/s transmission over $406 \mathrm{~km}$ of NDSF using mid-span spectral inversion by four-wave-mixing in a $2 \mathrm{~mm}$ long semiconductor optical amplifier," Electronics Letters 33, 879 (1997).

[11] M. Kuschnerov, F. N. Hauske, K. Piyawanno, B. Spinnler, M. S. Alfiad, A. Napoli, and B. Lankl, "DSP for coherent single-carrier receivers," Journal of Lightwave Technology 27, 3614-3622 (2009).

[12] X. Li, X. Chen, G. Goldfarb, Eduardo Mateo, I. Kim, F. Yaman, and G. Li, “Electronic post-compensation of WDM transmission impairments using coherent detection and digital signal processing," Opt. Express, 16, 880 (2008).

[13] D. Rafique, J. Zhao, and A. D. Ellis, "Digital back-propagation for spectrally efficient WDM 112 Gbit/s PM m-ary QAM transmission," Opt. Express 19, 5219-5224 (2011).

[14] C. Weber, C.-A. Bunge, and K. Petermann, "Fiber nonlinearities in systems using electronic predistortion of dispersion at 10 and $40 \mathrm{Gbit} / \mathrm{s}$," Journal of Lightwave Technology 27, 3654-3661 (2009).

[15] G. Goldfarb, M.G. Taylor, and G. Li, “Experimental demonstration of fiber impairment compensation using the split step infinite impulse response method," IEEE LEOS, ME3.1 (2008).

[16] D. Rafique and A. D. Ellis, "Impact of signal-ASE four-wave mixing on the effectiveness of digital back-propagation in $112 \mathrm{~Gb} / \mathrm{s}$ PM-QPSK systems," Opt. Express 19, 3449-3454 (2011).

[17] L.B. Du, and A. J. Lowery, "Improved single channel backpropagation for intra-channel fiber nonlinearity compensation in long-haul optical communication systems," Opt. Express 18, 17075-17088 (2010).

[18] L. Lei, Z. Tao, L. Dou, W. Yan, S. Oda, T. Tanimura, T. Hoshida, and J. C. Rasmussen, "Implementation Efficient Nonlinear Equalizer Based on Correlated Digital Backpropagation," OFC/NFOEC, OWW3 (2011).

[19] S. J. Savory, G. Gavioli, E. Torrengo, and P. Poggiolini, "Impact of Interchannel Nonlinearities on a Split-Step Intrachannel Nonlinear Equalizer," Photonics Technology Letters, IEEE 22, 673-675 (2010). 
[20] D. Rafique and A. D. Ellis, "Nonlinear Penalties in Dynamic Optical Networks Employing Autonomous Transponders," Photonics Technology Letters, IEEE 23, 1213-1215 (2011).

[21] D. Rafique, M. Mussolin, M. Forzati, J. Martensson, M.N. Chugtai, A.D. Ellis, “Compensation of intra-channel nonlinear fibre impairments using simplified digital backpropagation algorithm", Optics Express, Opt. Express 19, 9453-9460 (2011).

[22] C. S. Fludger, T. Duthel, D. vanden Borne, C. Schulien, E.-D. Schmidt, T. Wuth, J. Geyer, E. DeMan, G.-D. Khoe, and H. de Waardt,, "Coherent Equalization and POLMUX-RZ-DQPSK for Robust 100-GE Transmission," J. Lightwave Technol. 26, 64-72 (2008).

[23] T. Wuth, M. W. Chbat, and V. F. Kamalov, "Multi-rate (100G/40G/10G) Transport over deployed optical networks," OFC/NFOEC, NTuB3 (2008).

[24] W. Wei, Z. Lei, and Q. Dayou, "Wavelength-based sub-carrier multiplexing and grooming for optical networks bandwidth virtualization," OFC/NFOEC, PDP35 (2008)

[25] R. Peter, and C. Brandon, "Evolution to colorless and directionless ROADM architectures," OFC/NFOEC, NWE2 (2008).

[26] D. Rafique and A.D. Ellis "Nonlinear penalties in long-haul optical networks employing dynamic transponders," Optics Express 19, 9044-9049, (2011).

[27] S. Turitsyn, M. Sorokina, and S. Derevyanko, "Dispersion-dominated nonlinear fiberoptic channel," OpticsLetters 37, 2931-2933 (2012) .

[28] L.E. Nelson, A. H. Gnauck, R. I. Jopson, and A. R. Chraplyvy, “Cross-phase modulation resonances in wavelength-division-multiplexed lightwave transmission," ECOC, 309-310 (1998).

[29] D. Rafique, J. Zhao, and A. D. Ellis, "Digital back-propagation for spectrally efficient WDM 112 Gbit/s PM m-ary QAM transmission," Opt. Express 19, 5219-5224 (2011).

[30] D. Rafique and A.D. Ellis "Nonlinear and ROADM induced penalties in 28 Gbaud dynamic optical mesh networks employing electronic signal processing," Optics Express 19, 16739-16748, (2011).

[31] D. Rafique and A. D. Ellis, "Various Nonlinearity Mitigation Techniques Employing Optical and Electronic Approaches," Photonics Technology Letters, IEEE 23, 1838-1840 (2011).

[32] K. Roberts, "Digital signal processing for coherent optical communications: current state of the art and future challenges," SPPCOM, SPWC1 (2011).

[33] F. Heismann, "System requirements for WSS filter shape in cascaded ROADM networks," OFC/NFOEC, OThR1 (2010). 
[34] T. Otani, N. Antoniades, I. Roudas, and T. E. Stern, “Cascadability of passband-flattened arrayed waveguidegrating filters in WDM optical networks," Photonics Technology Letters 11, 1414-1416 (1999).

[35] M. Filer, and S. Tibuleac, "DWDM transmission at $10 \mathrm{~Gb} / \mathrm{s}$ and $40 \mathrm{~Gb} / \mathrm{s}$ using $25 \mathrm{GHz}$ grid and flexible-bandwidth ROADM," OFC/NFOEC, NThB3 (2011). 